\title{
Endogenous Market Structure, Occupational Choice, and Growth Cycles
}

\author{
Dimitrios Varvarigos \\ Department of Economics \\ University of Leicester \\ Telephone: ++44 (0) 1162522184 \\ Email: dv33@le.ac.uk
}

\author{
Maria José Gil-Moltó \\ Department of Economics \\ University of Sheffield \\ Telephone: +44 (0) 1142223416 \\ Email: m.j.gil-molto@sheffield.ac.uk
}

\footnotetext{
ฯ For their useful comments and suggestions, we would like to thank Vincenzo Denicolò; seminar participants at the University of Leicester, the University of Exeter, and the University of Sheffield; and participants of the 39th conference of the European Association of Research in Industrial Economics (Rome 2012), the 2013 Growth and Policy conference in Durham, and the $13^{\text {th }}$ conference of the Society for the Advancement of Economic Theory (Paris 2013), and the 2013 annual meeting of the Association of Southern European Economic Theorists (Bilbao).
} 
Running Head: "Endogenous Market Structure and Cycles"

Corresponding Author (please send proofs or any other communication to the following):

Dr Dimitrios Varvarigos

Department of Economics

University of Leicester

Astley Clarke Building

University Road

Leicester LE1 7RH

United Kingdom

Telephone: ++44 (0) 1162522184

Email: dv33@le.ac.uk 


\begin{abstract}
We model an industry that supplies intermediate goods in a growing economy. Agents can choose whether to provide labour or to become firm owners and compete in the industry. The idea that entry is determined through occupational choice has major implications for the economy's dynamics. Particularly, the results show that economic dynamics are governed by endogenous volatility in the determination of both the number of industry entrants and in the growth rate of output. Consequently, we argue that occupational choice and the structural characteristics of the endogenous market structure can act as both the impulse source and the propagation mechanism of economic fluctuations.
\end{abstract}

Keywords: Overlapping generations, Endogenous cycles, Market entry, Industry Dynamics JEL Classification: E32, L16 


\section{Introduction}

What are the fundamental sources behind economic fluctuations? The efforts to address this question have always been at the forefront of research in macroeconomics. Contrary to more conventional approaches that view exogenous (demand and/or supply) shocks as the initial impulse sources behind fluctuations in major economic variables, there is another strand of literature arguing that there is no reason to restrict attention to such exogenous processes as the generating causes of economic volatility. ${ }^{1}$ Instead, its impulse source may be embedded in the deep structural characteristics that shape the economy's dynamics and may lead economic variables to display fluctuations, either through damped oscillations; or through periodic orbits that are of a more permanent nature; or through stochastic economic fluctuations generated by purely extrinsic uncertainty (i.e., sunspots) rather than shocks to such fundamentals as preferences or technologies. Analyses on this strand of literature include the papers by Grandmont (1985); Benhabib and Nishimura (1985); Azariadis and Guesnerie (1986); Reichlin (1986); Azariadis and Smith (1996); Grandmont et al. (1998); Matsuyama (1999); Banerji et al. (2004); and Kaas and Zink (2007) among others. Our paper seeks to contribute to this strand of literature by offering a theory that complements the existing ones in enriching our current understanding on the extent to which endogenous forces can be propagated and manifest themselves in economic cycles.

\footnotetext{
${ }^{1}$ We refer to analyses that view economic fluctuations as only transitory or short-term phenomena, commonly known as 'business cycles'. The main idea is that various exogenous shocks represent the initial impulse sources whose effect is propagated and manifested in fluctuations of major economic variables. Different strands of literature, such as the real business cycle and the new-Keynesian approaches, have debated on both the impulse sources and the propagation mechanisms that lead to economic fluctuations.
} 
We are motivated by an emerging literature of research papers that incorporate both endogenous entry and strategic interactions among firms, as a means of enriching the potential propagation mechanisms in fully-fledged dynamic general equilibrium models that include exogenous shocks. ${ }^{2}$ The papers by Ghironi and Melitz (2005), Etro and Colciago (2010), Colciago and Etro (2010) and Bilbiie et al. (2012) analyse and discuss the ability of such frameworks in capturing stylised facts of key economic variables over the cycle. We also incorporate an endogenous market structure, taking the form of an industry whose firms produce and supply intermediate goods in our dynamic model. Rather than analysing how this structure can propagate the initial impact of an exogenous shock however, we argue that the structural characteristics that determine the equilibrium dynamics of the industry act as both the impulse source and the propagation mechanism that generates fluctuations in output growth. ${ }^{3}$ In this respect, our analysis is conceptually closer to the work undertaken by Chatterjee et al. (1993) and Dos Santos Ferreira and Lloyd-Braga (2005) who find that the dynamic equilibrium can converge to endogenous cycles, in models with imperfect competition and endogenous entry. In Chatterjee et al. (1993), a demand externality generates strategic complementarities in the decisions regarding market entry among different producers. As a result, the incentive of a potential entrant to create a firm and compete in the market is actually increasing in the existing number of firms, thus leading to multiple equilibria, as well as sunspots that are represented by a two-state Markov process. Dos Santos Ferreira and Lloyd-Braga (2005) build a model where households/workers and potential entrepreneurs are distinct in the sense that are born with predetermined abilities

\footnotetext{
${ }^{2}$ See Etro (2009) and the references therein for a more detailed discussion on this strand of literature.

${ }^{3}$ Other macroeconomic analyses that explicitly account for entry dynamics are, among others, those by Gil et al. (2013); Zeng (2013); and Sanders (2013).
} 
that deters them from choosing a different occupation, apart from the one that nature dictates to the group that they belong to. The households' labour supply is elastic while entrepreneurs face a fixed (exogenous) cost of entry in order to compete in the market of intermediate goods. The combination of these two factors affects the determination of the price mark-up whereas the latter impinges on the dynamics of capital accumulation. These dynamics generate indeterminacy, stochastic sunspot equilibria and deterministic cycles through Hopf bifurcations, even under circumstances that would rule out the existence such fluctuations in a perfectly competitive environment.

Similarly to these latter analyses, our model makes an explicit distinction between the different stages of an agent's lifetime, made possible by the OLG setting that we employ. The reason why the equilibrium number of competitors in the industry varies over time is radically different however. In particular, the dynamics of the industry in our paper rest on the following structural characteristics. First and foremost, the number of agents that choose to become intermediate good producers and join the industry, rather than becoming workers in the final goods sector, is determined through an occupational choice process. In other words, agents are not born into distinct groups whose occupational possibilities are restricted. This is a significant departure whose empirical relevance is evident from arguments such as the one by Yu et al. (2009) who state that "the decision to open a business reflects lifetime comparisons of anticipated earnings from self-employment with wage or salaried employment" (Yu et al. 2009; page 3). In terms of our model, occupational choice replaces the more familiar zero profit condition with a condition according to which agents compare the utility associated with a particular choice of occupation. Consequently, the implied (utility) cost of market entry is not fixed; instead, it varies with both the pre-existing and the anticipated future number of competitive firms - an outcome that has significant 
implications for the economy's dynamics as it will transpire in the main part of our paper. Secondly, contrary to labour, intermediate good production requires some specific training that delays the agent's entrance in the industry for the latter stage of her lifetime, thus allowing the number of market entrants to be effectively a state variable.

The combination of these characteristics in an OLG setting introduces rich dynamics with regards to the industry's structure. Particularly, the industry displays endogenous volatility; that is, fluctuations in market entry are not governed by the presence of exogenous shocks. Instead, they are manifested in either damped oscillations, or sunspot equilibria, or limit cycles. Damped oscillations and sunspots occur when the steady state equilibrium is locally stable (a sink) but also locally indeterminate; the latter when the conditions for stability are not satisfied (the equilibrium is a saddle point) and the dynamics can display flip - or period doubling - bifurcations. These cyclical trajectories rest on the strong non-monotonicities that pervade the dynamics of the industry. Despite the fact that technological progress is exogenous and firms do not contribute to any productivity-enhancing $R \& D$, these fluctuations generate endogenous cycles in the growth rate of output. These growth cycles are solely associated with the cyclical nature of entry and the corresponding variations in output that result from both the number of intermediate goods and the amount of labour. Again, growth cycles manifest themselves either through damped oscillations, or sunspots, or periodic orbits, depending on the corresponding dynamics for the intermediate goods industry - dynamics to which we alluded earlier.

Our premise that occupational choice is a significant element in the emergence of endogenously driven cycles in market entry is not a trivial issue. Realistically, people are not born with a predetermined career path; instead, their choice of occupation is one of the most important economic decisions that they make over their lifetime - a decision that, as we 
argued previously, researchers view as fundamental in the process of business formation. Our argument is that the elements that affect this choice are important in generating fluctuations in entry and consequently economic activity. Indeed, if we remove occupational choice from our framework and assume instead that agents are born with predetermined employment possibilities, then endogenous fluctuations would be automatically ruled out. In terms of evidence, Koellinger and Thurik (2012) provide empirical support for this argument. They measure entrepreneurial activity as the share self-employed individuals and owners/managers of businesses in the total labour force - suggestive of a choice between entrepreneurial activity and paid labour - and find evidence that fluctuations in entrepreneurship appear to cause fluctuations in GDP per capita. Further empirical support on the link between occupational choice and economic fluctuations is discussed in Carrasco (1999) who finds evidence that entry into self-employment is pro-cyclical. This is another characteristic of our model, since the different age structure of the individuals engaged in alternative occupations allows a positive contemporaneous relation between output, the number of intermediate good firms (the 'self-employed' of our model) and the amount of labour, despite the trade-off associated with occupational choice. Pro-cyclical entry is also a characteristic in Chatterjee et al. (1993) and Dos Santos Ferreira and Lloyd-Braga (2005) but the mechanisms and implications of our paper are much different.

At this point, we should emphasise that other papers have also identified the importance of occupational choice for economic outcomes. For example, Banerjee and Newman (1993) analyse its significance for the dynamics of income inequality and economic development. Rampini (2004) builds a model where different occupational opportunities vary in terms of both risk and return, and calibrates it in order to analyse the cyclical characteristics of entrepreneurial activities. Given the implications for indeterminacy, one of our results 
echoes the main implications of the analysis by Mino et al. (2005). They also use an overlapping generations setting to show that occupational choice can be responsible for dynamic indeterminacy. However, there are notable differences between their setting and ours. Firstly, they do not endogenise the number of firms that operate in a particular sector; instead, they assume that both sectors in the economy (producing consumption and investment goods) are perfectly competitive. Secondly, the occupational choice entails a decision on whether to become a skilled worker or remain unskilled - with both types of labour being imperfect substitutes in production. Therefore, the aim and implications of our paper differ significantly.

All in all, our main message adds to the current understanding that stems from existing theories on endogenous market structures within dynamic general equilibirium set-ups, as well as existing theories on endogenous volatility. With respect to the former, we show that the endogenous determination of industry dynamics is not only a stronger propagation mechanism; it may also represent the actual impulse source of growth cycles. With respect to the latter, we show that the combination of occupational choice and endogenous market structure can represent yet another important explanatory factor in the emergence of recurrent cycles in economic activity.

Despite the fact that our endeavour is to present a theoretical framework that offers qualitative implications, rather than quantitative ones, it should be noted that our results are not alien to empirical facts. For example, the data seems to support the idea that business cycles are not just short-lived phenomena. On the contrary, existing work (e.g., Comin and Gertler 2006) has offered evidence showing that cycles are relevant to lower frequencies as well - an outcome that corroborates with our model's OLG structure. Furthermore, there is evidence to suggest the existence of medium- and long-term oscillations in industrial activity 
(e.g., Geroski 1995; Keklik 2003; Baker and Agapiou 2006) in addition to the more commonly observed short-term movements related to the incidence of business cycles again, a fact which is in accordance with the main mechanism of our equilibrium results.

The remainder of our analysis is organised as follows. In Section 2 we lay down the basic set up of our economy. Section 3 derives the temporary equilibrium while Section 4 analyses and discusses the dynamic equilibrium and its implications. In Section 5 we conclude.

\section{The Economic Environment}

Time is discrete and indexed by $t=0,1,2, \ldots$. We consider an economy composed of a constant population of agents that belong to overlapping generations. Every period, a mass of $n>1$ agents is born and each of them lives for two periods - youth and old age. During their youth, agents are endowed with a unit of time which they can devote (inelastically) to one of the two available occupational opportunities. One choice is to be employed by perfectly competitive firms who produce the economy's final good. In this case, they receive the competitive salary $w_{t}$ for their labour services. Alternatively, they can devote their unit of time to some educational activity that will equip them with the ability to use managerial effort and produce units of a specific variety $j$ of an intermediate good when they are old. Intermediate goods are used by the firms that produce and supply the final good. We assume that, once made, occupational choices are irreversible.

The lifetime utility function of an agent born in period by $t$ is given by

$$
u_{j}^{t}=\left(c_{t, j}^{t}\right)^{1-\beta}\left(c_{t+1, j}^{t}\right)^{\beta}-\psi V\left(e_{t+1, j}\right) \text {, }
$$

where $c_{t, j}^{t}$ denotes the consumption of final goods during youth, $c_{t+1, j}^{t}$ denotes the consumption of final goods during old age and $\beta \in(0,1)$ is the relative weight attached to 
the utility accrued from consumption during old age. Furthermore, $e_{t+1, j}$ denotes effort and $V\left(e_{t+1, j}\right)$ is a continuous function that captures the disutility from effort and satisfies $V(0)=0$ and $V^{\prime}>0$. The parameter $\psi$ is a binomial indicator that takes the value $\psi=0$ if the agent is a worker and $\psi=1$ if the agent is an intermediate good producer. As this notation is important for the clarity of the subsequent analysis, it is important to note that the time superscript indicates the period in which the agent is born whereas the time subscript indicates the period in which an activity actually occurs. The subscript $j$ will be applicable only for producers of intermediate inputs; thus, it will be later removed from variables that are relevant to workers.

We assume that the final good is the numéraire. The production of this good is undertaken by a large mass (normalised to one) of perfectly competitive firms. These firms combine labour from young agents, denoted $L_{t}$, and all the available varieties of intermediate goods, each of them denoted $x_{t, j}$, to produce $y_{t}$ units of output according to

$$
y_{t}=A_{t}\left[N_{t}^{-\frac{1}{\theta-1}}\left(\sum_{j=1}^{N_{t}} x_{t, j}^{\frac{\theta-1}{\theta}}\right)^{\frac{\theta}{\theta-1}}\right]_{t}^{a} L_{t}^{1-a}
$$

where $a \in(0,1)$. The parameter $\theta>1$ is the elasticity of substitution between different varieties of intermediate goods and $N_{t}$ gives the number of these different varieties (see Dixit and Stiglitz 1977). ${ }^{4}$ Therefore, the latter variable is the number of entrants operating in

${ }^{4}$ The scale factor $N_{t}^{-1 /(\theta-1)}$ implies that, in a symmetric equilibrium, $N_{t}^{-1 /(\theta-1)}\left(\sum_{j=1}^{N_{t}} x_{t, j}^{(\theta-1) / \theta}\right)^{\theta /(\theta-1)}=N_{t} x_{t}$. 
the oligopolistic industry at time $t$. The variable $A_{t}$ denotes total factor productivity, which we assume to grow at a constant rate $g>0$ every period. Therefore,

$$
A_{t}=(1+g)^{t} A_{0}
$$

where the initial value $A_{0}>0$ is given. Note that, given the timing of events, the initial period's number of intermediate good firms is also exogenously given by $N_{0} \in(1, n)$.

The production of intermediate goods takes place under Bertrand competition among producers. Each of them uses her managerial effort and produces units of an intermediate good according to

$$
x_{t, j}=\gamma e_{t, j}, \quad \gamma>0 .
$$

Denoting the price of each intermediate good by $p_{t, j}$, the owner's revenue is given by $p_{t, j} x_{t, j}$. As we indicated above, the cost associated with the managerial activity is the effort/disutility cost characterised by the function $V(\cdot)$.

The process according to which agents choose their occupation involves the comparison of the lifetime utility that corresponds to being either a worker or an intermediate good producer. This problem will be formally solved at a later stage in our analysis. Now we will identify the pattern of optimal consumption choices made by each agent, taking her occupational choice as given.

We shall assume that this is a small open economy in the sense that individuals can save or borrow funds at the fixed interest rate $r>0 .{ }^{5}$ Furthermore, let us denote the present

\footnotetext{
${ }^{5}$ The assumption of a small open economy implies that domestic borrowing and lending cannot affect the world interest rate $r$.
} 
value of an agent's lifetime income by $i_{t}$. Given these, we can write her lifetime budget constraint as

$$
c_{t, j}^{t}+\frac{c_{t+1, j}^{t}}{1+r}=i_{t}
$$

Substituting (5) in (1), we can calculate $\frac{\partial u_{j}^{t}}{\partial c_{t+1, j}^{t}}=0$ in order to derive

$$
c_{t+1, j}^{t}=\beta(1+r) i_{i},
$$

i.e., the demand for consumption goods during old age. Combining (6) and (5), we can derive the corresponding demand function for goods consumed during youth. This is given by

$$
c_{t, j}^{t}=(1-\beta) i_{t} .
$$

As expected, an individual's consumption expenditures during each period are proportional to her lifetime income (in present value terms). The relative utility weight $\beta$ is crucial in determining what proportion of lifetime income will be devoted to the satisfaction of consumption needs in either youth or old age.

Now, let us consider each group of agents separately, beginning with the workers for whom the lifetime income corresponds to the one accruing from their labour services, i.e., $i_{t}=w_{t}$. Given the previous discussion and results, for those young agents whose choice is to provide labour we have $\psi=0, c_{t}^{t, \text { worker }}=(1-\beta) w_{t}$ and $c_{t+1}^{t, \text { worker }}=\beta(1+r) w_{t}$, meaning that each worker saves an amount $s_{t}=\beta w_{t}$ during her youth. Therefore, we can use (1) to write the lifetime utility of a worker born in period $t$ as

$$
u^{t, \text { worker }}=\zeta w_{t},
$$

where $\zeta$ is a composite parameter term given by 


$$
\zeta \equiv(1-\beta)^{(1-\beta)} \beta^{\beta}(1+r)^{\beta} .
$$

For intermediate good producers, however, the equilibrium characteristics are different.

Particularly, their lifetime income (in present value terms) equals $i_{t}=\frac{p_{t+1, j} x_{t+1, j}}{1+r}$. Therefore, those who decide to be intermediate good producers have $\psi=1$, $c_{t, j}^{t, \text { producer }}=(1-\beta) \frac{p_{t+1, j} x_{t+1, j}}{1+r}$ and $c_{t+1, j}^{t, \text { producer }}=\beta p_{t+1, j} x_{t+1, j}$, meaning that each of them borrows the amount $b_{t, j}=(1-\beta) \frac{p_{t+1, j} x_{t+1, j}}{1+r}$ during her youth. ${ }^{6}$ Using these results in (1), we get the lifetime utility of a producer born in period $t$ as

$$
u_{j}^{t, \text { producer }}=\not p_{t+1, j} x_{t+1, j}-V\left(e_{t+1, j}\right) \text {, }
$$

where $z$ is a composite parameter term given by

$$
z \equiv(1-\beta)^{(1-\beta)} \beta^{\beta}(1+r)^{\beta-1}
$$

With this discussion we have completed the basic set up of our economy. In the sections that follow we derive the economy's temporary and dynamic equilibrium, with particular emphasis on the dynamics of the intermediate goods industry.

\section{Temporary Equilibrium}

For the producers of final goods, profit maximisation implies that each input earns its marginal product. In terms of labour income, we have

\footnotetext{
${ }^{6}$ Note that the implied cost of the activity that equipes an agent with the ability to become an intermediate good producer, corresponds to the foregone labour income. We could have allowed an additional direct cost, taking the form of a proportion of (the present value of) her second period earnings. This would have introduced an additional scale factor to the model, without altering our results.
} 


$$
w_{t}=(1-a) A_{t}\left[N_{t}^{-\frac{1}{\theta-1}}\left(\sum_{j=1}^{N_{t}} x_{t, j}^{\frac{\theta-1}{\theta}}\right]^{\frac{\theta}{\theta-1}}\right]^{a} L_{t}^{-a}=(1-a) \frac{y_{t}}{L_{t}} .
$$

For intermediate goods we have

$$
p_{t, j}=A_{t} L_{t}^{1-a} a N_{t}^{-\frac{a}{\theta-1}}\left[\left(\sum_{j=1}^{N_{t}} x_{t, j}^{\frac{\theta-1}{\theta}}\right)^{\frac{\theta}{\theta-1}}\right]^{a-1}\left(\sum_{j=1}^{N_{t}} x_{t, j}^{\frac{\theta-1}{\theta}}\right)^{\frac{\theta}{\theta-1}-1} x_{t, j}^{\frac{\theta-1}{\theta}-1} .
$$

Multiplying both sides of (13) by $x_{t, j}$ and summing over all $j$ 's, we can get

$$
\sum_{j=1}^{N_{t}} p_{t, j} x_{t, j}=a A_{t}\left[N_{t}^{-\frac{1}{\theta-1}}\left(\sum_{j=1}^{N_{t}} x_{t, j}^{\frac{\theta-1}{\theta}}\right)^{\frac{\theta}{\theta-1}}\right]^{a} L_{t}^{1-a} .
$$

We can combine Equations (13) and (14) and exercise some straightforward, but tedious, algebra to derive the demand function for an intermediate good. This is given by

$$
x_{t, j}=\left(\frac{p_{t, j}}{P_{t}}\right)^{-\theta} \frac{X_{t}}{N_{t}}
$$

where

$$
X_{t}=N_{t}^{-\frac{1}{\theta-1}}\left(\sum_{j=1}^{N_{t}} x_{t, j}^{\frac{\theta-1}{\theta}}\right)^{\frac{\theta}{\theta-1}}
$$

Furthermore, using $\sum_{j=1}^{N_{t}} p_{t, j} x_{t, j}=P_{t} X_{t}$, the price index is given by

$$
P_{t}=\left(\frac{1}{N_{t}} \sum_{j=1}^{N_{t}} p_{t, j}^{1-\theta}\right)^{\frac{1}{1-\theta}} .
$$

The result in (15) is nothing else than the familiar inverse demand function in models with a constant elasticity of substitution between different varieties of goods (Dixit and Stiglitz 1977). In other words, the share of product $j$ in the overall demand for intermediate 
inputs is inversely related to its relative price. This effect is more pronounced with higher values of $\theta$, i.e., if different varieties are less heterogeneous, thus more easily substitutable.

Now let us consider the equilibrium in the labour, the financial and the final goods markets. With respect to the former, the demand for labour by firms $\left(L_{t}\right)$ must be equal to the supply of labour by young agents. Recall that in period $t$, out of the total population mass of $n$, some agents will decide to set up firms and produce intermediate goods in period $t+1$. The number of these agents is $N_{t+1}$. Therefore, the labour market equilibrium is

$$
L_{t}=n-N_{t+1} .
$$

As for the financial market, let us define aggregate debt (denoted $d_{t}$ ) as the difference between borrowing and saving, i.e.,

$$
d_{t}=\sum_{j=1}^{N_{t+1}} b_{t, j}-L_{t} s_{t}
$$

Naturally, debt evolves according to

$$
d_{t}=(1+r) d_{t-1}+C_{t}-y_{t},
$$

where $C_{t}$ is the aggregate consumption expenditure in period $t^{7}$ Furthermore, using the expressions in (2), (12) and (14) it becomes clear that the constant returns technology implies that

$$
y_{t}=L_{t} w_{t}+\sum_{j=1}^{N_{t}} p_{t, j} x_{t, j} .
$$

Combining (19)-(21), we can write Equation (20) as

\footnotetext{
${ }^{7}$ Note that aggregate debt can be negative, in which case Equation (20) is the accumulation of assets in the economy.
} 


$$
\begin{aligned}
& \sum_{j=1}^{N_{t+1}} b_{t, j}-L_{t} s_{t}=(1+r)\left(\sum_{j=1}^{N_{t}} b_{t-1, j}-L_{t-1} s_{t-1}\right)+C_{t}-L_{t} w_{t}-\sum_{j=1}^{N_{t}} p_{t, j} x_{t, j} \Leftrightarrow \\
& \sum_{j=1}^{N_{t+1}} b_{t, j}+L_{t}\left(w_{t}-s_{t}\right)+\left[\sum_{j=1}^{N_{t}} p_{t, j} x_{t, j}-(1+r) \sum_{j=1}^{N_{t}} b_{t-1, j}\right]+L_{t-1}(1+r) s_{t-1}=C_{t} .
\end{aligned}
$$

We can use previous results to rewrite Equation (22) as

$$
\sum_{j=1}^{N_{t+1}} c_{t, j}^{t, \text { producer }}+L_{t} c_{t}^{t, \text { worker }}+\sum_{j=1}^{N_{t}} c_{t, j}^{t-1, \text { producer }}+L_{t-1} c_{t}^{t-1, \text { worker }}=C_{t},
$$

an expression that corresponds to the equilibrium in the goods market.

Now, we can use $\sum_{j=1}^{N_{t}} p_{t, j} x_{t, j}=P_{t} X_{t}$, (12), (17) and (21) in (15) to write the demand function for the intermediate good as

$$
x_{t, j}=\frac{p_{t, j}^{-\theta}}{\sum_{j=1}^{N_{t}} p_{t, j}^{1-\theta}} a y_{t}
$$

The result in (24) is more explicit on the interactions in the pricing decisions made by competing firms. It can be used to solve the utility maximisation problem of an agent who produces intermediate goods. To this purpose, it will be useful to specify a functional form for the effort cost component $V\left(e_{t+1, j}\right)$. For this reason, and to ensure analytical tractability, we specify

$$
V\left(e_{t+1, j}\right)=m e_{t+1, j}, \quad m>0 .
$$

Writing Equation (24) in terms of period $t+1$ and substituting it together with (4) and (25) in (10), allows us to write the utility function of the producer $j$ as

$$
u_{j}^{t, \text { producer }}=\left(2 p_{t+1, j}-\frac{m}{\gamma}\right) \frac{p_{t+1, j}^{-\theta}}{\sum_{j=1}^{N_{t+1}} p_{t+1, j}^{1-\theta}} a y_{t+1}
$$


Given that firm owners operate under Bertrand competition, their objective is to choose the price of their products in order to maximise their lifetime utility. In other words, their objective is

$$
\max _{p_{t+1, j}}\left\{\left(2 p_{t+1, j}-\frac{m}{\gamma}\right) \frac{p_{t+1, j}^{-\theta}}{\sum_{j=1}^{N_{t+1}} p_{t+1, j}^{1-\theta}} a y_{t+1}\right\} .
$$

After some straightforward algebra, it can be shown that the solution to this problem leads to a symmetric equilibrium for which

$$
p_{t+1, j}=p_{t+1} \text { and } x_{t+1, j}=x_{t+1} \forall j \text {, }
$$

where the optimal price equals

$$
p_{t+1}=\frac{m}{\gamma \Sigma} \frac{\left[\theta\left(N_{t+1}-1\right)+1\right]}{(\theta-1)\left(N_{t+1}-1\right)}
$$

In addition, given (17) and (22), the equilibrium quantity of the intermediate good by each entrepreneur is

$$
x_{t+1}=\frac{a y_{t+1}}{N_{t+1}} \frac{\gamma z}{m} \frac{(\theta-1)\left(N_{t+1}-1\right)}{\left[\theta\left(N_{t+1}-1\right)+1\right]} .
$$

The result in Equation (30) resembles the familiar condition according to which the price is set as a mark-up over the marginal cost of production. In this case, each producer sets a mark-up over the marginal utility cost of producing the intermediate good, since one unit of production requires a utility cost of $m / \gamma$ units of effort. Naturally, the mark-up is decreasing in the number of producers because the latter implies a more intensely competitive environment. Additionally, the mark-up is also decreasing in $\theta$ because higher values of this parameter increase the degree of substitutability between different varieties of intermediate goods - yet another structural characteristic that enhances the degree of 
competition. From Equation (30), we can see that the inverse demand function implies that the components that reduce the relative price of the input increase its share on aggregate demand.

The solutions above allow us to rewrite the utility of an intermediate good producer, after substituting (28) and (29) in (26), as follows:

$$
u^{t, \text { producer }}=\frac{z a y_{t+1}}{\theta\left(N_{t+1}-1\right)+1} .
$$

With this result at hand, we can now turn our attention to the occupational choice problem of an agent who is young in period $t$.

\subsection{Occupational Choice}

Our purpose in this section is to determine how many agents will decide to become suppliers of intermediate inputs. Obviously, the equilibrium condition requires that an agent born in $t$ should be indifferent between the two different occupational opportunities. Formally, a condition that needs to hold in equilibrium is

$$
u^{t, \text { producer }}=u^{t, \text { worker }} \text {, }
$$

or, after utilising (8), (12) and (31),

$$
\frac{z a y_{t+1}}{\theta\left(N_{t+1}-1\right)+1}=\frac{\zeta(1-a) y_{t}}{L_{t}} \text {. }
$$

We can manipulate algebraically the expression in (33) even further. First, we can use the symmetry condition (Eq. 28) in (2) to get

$$
y_{t}=A_{t}\left(N_{t} x_{t}\right)^{a} L_{t}^{1-a} .
$$

Next, we can use (30) to get 


$$
N_{t+1} x_{t+1}=a y_{t+1} \frac{\gamma z}{m} \frac{(\theta-1)\left(N_{t+1}-1\right)}{\left[\theta\left(N_{t+1}-1\right)+1\right]} \Leftrightarrow N_{t} x_{t}=a y_{t} \frac{\gamma z}{m} \frac{(\theta-1)\left(N_{t}-1\right)}{\left[\theta\left(N_{t}-1\right)+1\right]}
$$

Further substitution of (35) in (34) allows us to write

$$
y_{t}=A_{t}^{\frac{1}{1-a}}\left\{\frac{\operatorname{a\gamma z}}{m} \frac{(\theta-1)\left(N_{t}-1\right)}{\left[\theta\left(N_{t}-1\right)+1\right]}\right\}^{\frac{a}{1-a}} L_{t} .
$$

Finally, we can use (18) and (36) in (26), and rearrange to get

$$
\frac{n-N_{t+2}^{E}}{\theta\left(N_{t+1}-1\right)+1}=\frac{(1-a)(1+r)}{a(1+g)^{1 /(1-a)}}\left\{\frac{\left[\theta\left(N_{t+1}-1\right)+1\right]}{\left(N_{t+1}-1\right)} \frac{\left(N_{t}-1\right)}{\left[\theta\left(N_{t}-1\right)+1\right]}\right\}^{a /(1-a)},
$$

where $1+g=\frac{A_{t+1}}{A_{t}}$ is derived by alluding to (3). Note that the superscript in $N_{t+2}^{E}$ denotes the expectation formed on this variable.

The result in Equation (37) is the most important in our set-up. It implies that the determination of the equilibrium number of firms in the intermediate goods industry is not a static one. Instead, there will be some transitional dynamics as the number of producers converges to its long-run equilibrium. Particularly, we can see that the equilibrium number of firms in any given period depends on both the predetermined number of firms from the previous period and the expectation on the number of firms that will be active during the next period. Note that the endogenous occupational choice is critical for these dynamics. It is exactly because of this choice that the determination of $N_{t+1}$ is related to the previous period's demand conditions (and thus $N_{t}$ ) and the next period's labour market equilibrium (therefore $N_{t+2}^{E}$ ).

The intuition for these effects is as follows. If the existing number of intermediate good firms is large, then the overall amount of intermediate goods, and therefore the marginal product of labour, will be higher. This increases the equilibrium wage and thus the relative 
benefit from the utility of being a worker when young, rather than setting up a firm when old. ${ }^{8}$ Now suppose that, while forming their occupational choice, the current young expect that the future number of firms in the intermediate goods industry will be high. For them, this implies that the amount of labour, and therefore total demand in the next period will be relatively low. Thus, the relative utility benefit of being a firm owner when old, rather than a worker when young, is reduced because the expectation of lower future demand for final goods will have corresponding repercussions in terms of reduced future demand for intermediate goods as well. Consequently, a reduced number of individuals out of the current young will opt for the choice of becoming intermediate good producers.

\section{Dynamic Equilibrium}

The remainder of our analysis will focus on the dynamics of the industry that produces intermediate goods. In what follows, we consider the economy's perfect foresight dynamics, i.e., equilibrium trajectories that satisfy $N_{t+2}^{E}=N_{t+2}$.

\subsection{The Steady State}

We can obtain the stationary equilibrium for the number of intermediate good firms, after substituting $\quad N_{t+2}^{E}=N_{t+2}$ in (37) and using the steady state condition $N_{t+2}=N_{t+1}=N_{t}=\hat{N}$. This procedure will eventually allow us to derive

\footnotetext{
8 According to Parker (2009), this 'wage-effect' is an empirically important determinant when individuals choose whether to open a business or seek paid employment.
} 
Proposition 1. Suppose $n>1+\delta$ where $\delta=(1-a)(1+r) / a(1+g)^{1 /(1-a)}$. Then there exists a unique steady state equilibrium $\hat{N} \in(1, n)$ such that

$$
\hat{N}=\frac{n+(\theta-1)(1-a)(1+r) / a(1+g)^{1 /(1-a)}}{1+\theta(1-a)(1+r) / a(1+g)^{1 /(1-a)}}
$$

As long as the steady state solution is asymptotically stable, then for any predetermined $N_{0} \in(1, n)$ the equilibrium number of producers will eventually converge to $\hat{N}$ in the longrun. Later, we are going to formally characterise the conditions for the (local) stability of this equilibrium. For now, it is instructive to undertake some comparative statics to identify the effects of the economy's structural parameters on the steady state number of firms competing in the intermediate goods industry. This is a task that can be easily undertaken through the use of Equation (38). The results can be summarised in

Proposition 2. The long-run equilibrium number of firms in the intermediate goods industry is:

i. Increasing in the growth rate of total factor productivity $(g)$;

ii. Decreasing in the relative share of labour income $(1-a)$, the world interest rate $(r)$, and the degree of substitutability between different varieties of intermediate products $(\theta)$.

The economic interpretation for these results is as follows. A permanent increase in the growth rate causes future demand to become even higher compared to current demand because of the increase of the economy's resources. This effect boosts the relative utility benefit of becoming a firm owner, with corresponding implications for the occupational choices made by young agents. An increase in the relative share of labour income will 
motivate more agents to work for final goods firms, as the income earned from activities in the intermediate goods industry becomes relatively low. The utility benefit of such activities is also impeded in an industry where goods are less heterogeneous. Finally, when individuals face a higher borrowing rate, then they have a lower incentive to opt for the occupation which renders borrowing necessary in order to finance the first period's consumption needs - in this case, firm ownership.

\subsection{Transitional Dynamics}

Let us use $N_{t+2}^{E}=N_{t+2}$ in (37) and solve the resulting expression for $N_{t+2}$. Eventually, we get

$$
N_{t+2}=n-\frac{(1-a)(1+r)}{a(1+g)^{1 /(1-a)}} \frac{\left[\theta\left(N_{t+1}-1\right)+1\right]^{1 /(1-a)}}{\left(N_{t+1}-1\right)^{a /(1-a)}}\left[\frac{N_{t}-1}{\theta\left(N_{t}-1\right)+1}\right]^{a /(1-a)}=F\left(N_{t+1}, N_{t}\right)
$$

As we can see, the dynamics of the intermediate goods industry are characterised by a nonlinear, second-order difference equation in terms of the industry's size (i.e., the number of agents who compete in the industry).

One way to analyse the transition equation in (39) is to define $Z_{t}=N_{t+1}$ and treat the dynamics as being generated by the following system of first-order difference equations:

$$
\begin{gathered}
Z_{t+1}=F\left(Z_{t}, N_{t}\right)=n-\delta \frac{\left[\theta\left(Z_{t}-1\right)+1\right]^{1 /(1-a)}}{\left(Z_{t}-1\right)^{a /(1-a)}}\left[\frac{N_{t}-1}{\theta\left(N_{t}-1\right)+1}\right]^{a /(1-a)}, \\
N_{t+1}=H\left(Z_{t}, N_{t}\right)=Z_{t},
\end{gathered}
$$

where $N_{0}, Z_{0} \in(1, n)$ are taken as the initial conditions and the steady state satisfies $\hat{Z}=\hat{N}$ and $\delta$ is defined in Proposition 1. The Jacobian matrix associated with the planar system of Equations (40)-(41) is 


$$
\left(\begin{array}{ll}
F_{Z_{t}}(\hat{Z}, \hat{N}) & F_{N_{t}}(\hat{Z}, \hat{N}) \\
H_{Z_{t}}(\hat{Z}, \hat{N}) & H_{N_{t}}(\hat{Z}, \hat{N})
\end{array}\right)
$$

where $\hat{N}=\hat{Z}$ is given in (38). Furthermore, the eigenvalues are the roots of the polynomial $\lambda^{2}-T \lambda+D$, i.e.,

$$
\lambda_{1}=\frac{T-\sqrt{T^{2}-4 D}}{2} \text { and } \lambda_{2}=\frac{T+\sqrt{T^{2}-4 D}}{2} \text {, }
$$

where

$$
T=F_{Z_{t}}(\hat{Z}, \hat{N})+H_{N_{t}}(\hat{Z}, \hat{N})
$$

and

$$
D=F_{Z_{t}}(\hat{Z}, \hat{N}) H_{N_{t}}(\hat{Z}, \hat{N})-F_{N_{t}}(\hat{Z}, \hat{N}) H_{Z_{t}}(\hat{Z}, \hat{N})
$$

are respectively the trace and the determinant of the matrix. As it is well known (Azariadis 1993; Galor 2007) the eigenvalues can be used to check the stability of the steady state solution and to trace the transitional dynamics towards it. Later, it will transpire that, under different conditions, $\hat{N}$ can be either (locally) stable or unstable. For now, we will focus our attention to a case whereby the steady state equilibrium characterised by (38) is actually stable, while the possible implications that arise in the scenario where $\hat{N}$ is unstable will be discussed subsequently.

Let us begin by defining $\Xi(\delta) \equiv \delta+\frac{\delta a(1+\delta \theta)}{1-a} \frac{2}{\delta \theta-1}$ and $\tilde{\delta}$ such that $\Xi(\tilde{\delta})=n-1$.

Furthermore, the analysis that follows will be making use of the following assumptions:

Assumption 1. $n>1+2\left(1+\frac{3 a}{1-a}\right)$ 


\section{Assumption 2. $\delta \theta \leq 2$.}

Both assumptions are employed to make the analysis of the transitional dynamics more precise, clear and sharply focused. Firstly, Assumption 2 is sufficient to guarantee that, at least for some range of parameter values, the steady state equilibrium will be stable. Secondly, we want to focus solely on the emergence of flip (period doubling) bifurcations. In this respect, Assumption 1 is sufficient to guarantee that the eigenvalues of the dynamical system in (40)-(41) are real numbers. Finally, we wish to note that the proofs to the subsequent results are relegated to the Appendix.

The sufficient conditions for stability are formally described in

Lemma 1. If either (i) $\delta \theta \leq 1$ or (ii) $\delta \theta \in(1,2]$ and $\delta<\tilde{\delta}$, then the steady state solution $\hat{N}$ is locally stable. In other words, the dynamics starting from an initial value $N_{0} \in(1, n)$ will eventually converge to $\hat{N}$.

With respect to output, once the industry converges to its steady state, the production of final goods will converge to a balanced growth path. Along this path, output will grow at a constant rate that is proportional to the growth rate of total factor productivity. It is straightforward to use Equations (18) and (36) and the result of Lemma 1 to establish that

$$
\lim _{t \rightarrow \infty}\left(\frac{y_{t+1}}{y_{t}}-1\right)=(1+g)^{\frac{1}{1-a}}-1 \text {. }
$$

Nevertheless, during the transition to the balanced growth path, the dynamics of output will also be (partially) dictated by the transitional dynamics of the intermediate goods 
industry. A technical condition that can facilitate a better understanding of how the intermediate goods industry evolves over time is given by

Lemma 2. As long as the conditions for stability that are summarised in Lemma 1 hold, both eigenvalues are negative, i.e., $\lambda_{1}, \lambda_{2} \in(-1,0)$.

Using Lemma 2 we can characterise the transitional behaviour of the economy through

Proposition 3. Given Lemma 2, the number of firms in the intermediate goods industry converges to its long-term equilibrium through cycles. Consequently, output growth displays fluctuations as it converges to the balanced growth path.

Recall that the number of producers in any given period is affected by both the predetermined number of producers from the previous period and the expectation on the number of producers that will be active in the future. The manner and direction of these effects, both discussed at an earlier point of our analysis, render the result of Proposition 3 to be a quite intuitive one. For example, consider a situation where the existing number of intermediate good producers is low relative to the steady state. For the current young agents, the incentive to opt for market entry when old is enhanced because the marginal product of labour (and therefore the wage) is currently low. As a result, an increased fraction of the current young will choose to become firm owners and compete in the intermediate goods industry when they become old. However, for this to happen they also need to expect that, next period, a lower fraction of the future generation's agents will decide to become producers because this will increase labour, and therefore aggregate demand during the 
period where producers will reap the benefits of their activity. The mechanism that we described previously does verify this expectation, hence granting an even greater incentive to the agents for setting up intermediate good firms. Furthermore, it explains why both the size of the intermediate goods industry and output growth converge to their long-run equilibrium through cycles.

For illustrative purposes, in what follows we will analyse the transition equation in (39) numerically, making sure to choose parameter values that render the solution in (38) stable, hence a meaningful one. We should emphasise, however, that we undertake these numerical simulations solely as a means to illustrate the transitional behaviour of the economy. The focus of our analysis is still purely qualitative, hence it is neither our intention nor do we claim any attempt to offer a quantitative match of key moments from stylised facts.

For the baseline parameter values, we choose $a=0.5, g=0.32, r=0.3$ and $\theta=1.3$, while the total population is set to $n=100 .^{9}$ The initial values are $N_{0}=15$ and $N_{1}=85-$ recalling that $N_{1}$ corresponds to $Z_{0}$ in (41). In Figure 1 we see the transitional dynamics for the intermediate goods industry, based on this simulation. Given the numerical example, the steady state value for $\hat{N}$ is roughly 51 (50.8764 to be precise) and the industry converges to this equilibrium. Nevertheless, this convergence is clearly non-monotonic. Instead, convergence takes place through damped oscillations, or cycles, during which the number of firms takes values above and below the stationary value as the industry approaches towards it. In Figure 2 we use the same baseline parameter values, in addition to $A_{0}=10$, to simulate

\footnotetext{
${ }^{9}$ It can be easily established that these parameter values lie on the permissible range that guarantees stability according to Lemma 1.
} 
the movements of the growth rate of output, $\frac{y_{t+1}}{y_{t}}-1$. Again, we can see that, due to fluctuations that occupational choice generates in the determination of the number of producers each period, output converges to its balanced growth path (with a growth rate of 0.7424) through cycles. Note however that these fluctuations are not due to the fact that the intermediate goods industry is associated with some type of $\mathrm{R} \& \mathrm{D}$ that increases the rate of technological progress endogenously. Instead, they are purely associated with variations in output that result from the cyclical nature of $N_{t}$ and the corresponding variations in both the number of intermediate goods and the labour input.

[INSERT FIGURE 1 HERE]

[INSERT FIGURE 2 HERE]

\subsection{Indeterminacy and Sunspot Equilibria}

Before we proceed to the analysis of limit cycles, we will discuss the possibility of indeterminacy in the transitional dynamics of the economy. As we have seen from the second order transition equation in (39), or the equivalent dynamical system in (40)-(41), the transitional dynamics are traced after we consider two initial values $N_{0}$ and $Z_{0}$ - the latter corresponding to $N_{1}$. Nevertheless, while $N_{0}$ is indeed predetermined, this is not the case for $N_{1}$. Instead, taking the value of $N_{0}$ as given, $N_{1}$ reflects an equilibrium formed on an expectation about $N_{2}$ and so on. In other words, the stability of the steady state equilibrium $\hat{N}$ implies that, for the same $N_{0} \in(1, n)$, there are certainly more than one trajectories that 
are consistent with the economy's convergence to the steady state. In other words, economies that are identical both in terms of structural parameters and predetermined conditions may display very different equilibrium characteristics for a large part of their transition towards the common steady state.

Nevertheless, the aforementioned arguments - indicative of local indeterminacy - have more implications for the evolution of output. Particularly, the self-fulfilling nature of one of the mechanisms that permeates occupational choice implies that, in addition to the stationary equilibrium derived in (38), the economy's dynamics may also be characterised by stochastic cycles due to sunspots. These cycles are not driven by shocks to any of the model's fundamental parameters of preferences or technologies. Instead, they manifest the element of extrinsic uncertainty inherent to the fact that individuals choose their occupation (labour or intermediate goods production), partially based on an expectation about future outcomes. Their choice however is by itself conducive to the actual realisation of those outcomes. Indeed, previously we indicated that the incentive of agents to earn their lifetime income through intermediate goods production is increased when they expect that market entry will be reduced in the period afterwards. Due to the fact that current market entry is inversely related to the predetermined number of firms, these expectations can become self-fulfilling. If more agents join the industry now, then the future market entry will actually be relatively low, thus verifying their initial expectation. We can summarise these implication through

Proposition 4. As long as the conditions for stability that are summarised in Lemma 1 bold, then there exist sunspot equilibria in the neighbourbood of the stationary solution $\hat{N}$. 
A formal proof for the previous result appears in Woodford (1986) and Grandmont et al. (1998) - the former for the case of a second-order scalar system with only one predetermined value and the latter for a planar system of two state variables in which only one of them is predetermined. The existence of sunspots represents one type of permanent economic fluctuations in our model. In the subsequent section, we show that there may also be another type of fluctuations - in that case, not stochastic but deterministic - which occur as a result of period doubling bifurcations that result in oscillations, similar to the ones identified in Proposition 3, but at the same time rather different in the sense that these oscillations will be permanent.

\subsection{Periodic Equilibrium}

So far, we have seen scenarios in which oscillations in economic variables are not permanent - an outcome related to our restriction on conditions that guarantee the stability of the steady state. Nevertheless, it will also be interesting to examine the possibilities that arise when the steady state in (38) does not satisfy these stability conditions. This may happen in circumstances that are described in

Lemma 3. Suppose that $\delta \theta \in(1,2]$ and $\delta>\tilde{\delta}$. In this case, the two eigenvalues $\lambda_{1}$ and $\lambda_{2}$ satisfy $0>\lambda_{2}>-1>\lambda_{1}$. Therefore, the steady state solution $\hat{N}$ is a saddle point.

The saddle point property of the steady state implies that, for given $N_{0}$, there is only one corresponding $N_{1}\left(=Z_{0}\right)$ such that the industry dynamics follow a path of convergence towards $\hat{N}(=\hat{Z})$. All other paths will diverge away from this point. Now, recall that the 
dynamics are traced after we consider two initial values $N_{0}$ and $N_{1}=\left(Z_{0}\right)$ from which only $N_{0}$ is predetermined. This implies that we can rule out some divergent paths because they are clearly not optimal: as $N_{t}$ will at some point approach either 1 or $n$, output and consumption will become equal to zero. Nevertheless, there are paths that although not converging towards $\hat{N}$, there is no reason why they should be ruled out. These paths entail the presence of a periodic equilibrium or limit cycles. We will use the previous numerical example to illustrate such cases, bearing in mind that parameter values must satisfy the conditions summarised in Lemma 3.

In the baseline numerical example, we set the TFP growth factor equal to $g=0.26$. Doing so, the simulation indicates that the steady state $\hat{N}$ falls to roughly 49 (the exact number is 48.55687), but this is an unstable solution. Instead, the dynamics of entry converge to a 2-period cycle equal to $\left\{N^{1}, N^{2}\right\}=\{83.02957452,15.51242096\}$ that corresponds to a 2-period cycle for the growth rate $\{0.656219559,0.521823448\}$. This periodic equilibrium is in fact robust to changes in the initial conditions - an outcome that, together with the fact that they surround a unique but unstable solution, indicates that the 2 period cycle is stable. Cycles with two periodic solutions appear as we reduce $g$ even further, until at some point we observe that 2-period cycles become unstable and replaced by the emergence of a stable 4-period cycle. For example, setting $g=0.205$ leads to an unstable steady state $\hat{N}=46.33716$ and a 4-period cycle $\left\{N^{1}, N^{2}, N^{3}, N^{4}\right\}=\{89.6690622$, $4.97178912,93.46991857,8.330862453\}$ that corresponds to a 4-period cycle for the growth 
rate $\{0.590587824,0.226986784,0.718944528,0.325064367\} .{ }^{10}$ Reducing $g$ even more leads to the emergence of cycles of period-8 (e.g., for $g=0.199$ ), period-16 (e.g., for $g=0.1983$ ) and so on, suggesting that the economy's dynamics undergo a "period-doubling route to chaos" (Devaney 2003). Indeed, reducing the TFP growth factor to 0.185 generates cycles that are clearly aperiodic. Figure 3 provides the simulated graphs for all the examples that we offered above.

It is possible to generalise the implications offered by these numerical examples. We can start with

Lemma 4. Suppose that $\delta \theta \in(1,2]$. The dynamical system of (40) and (41) undergoes a flip (period doubling) bifurcation at $\delta=\tilde{\delta}$. Hence, there exist stable limit cycles.

Given this, we can characterise the dynamics in this case through

Proposition 5. Under the conditions in Lemmas 3 and 4, fluctuations in the number of intermediate good firms can become permanent. Therefore, output may not converge to its balanced growth path; instead it will fluctuate permanently around it.

Recall that $\delta$ is a composite parameter term that is negatively related to $g$. Given Lemma 4 , it is not difficult to understand why our previous simulations revealed that reductions of the TFP growth factor generate period doubling bifurcations. In terms of intuition, we can

\footnotetext{
${ }^{10}$ In these examples, we have ignored the discrete nature of $N_{t}$. The reader may approximate the appropriate value by using the closest integer.
} 
allude to the forces of industry dynamics that we described previously. Now however, the impact of non-monotonicities is strong enough so that cycles do not dissipate over time. On the contrary, they become a permanent characteristic of the industry's dynamics and consequently, the evolution of output. These fluctuations do not rest on any exogenous shocks. Instead, both the impulse source and the propagation mechanism lie with the structural characteristics of the economic environment. In particular, the occupational choice is the source of non-monotonicities that generate fluctuations and propagate them into fluctuations of output growth.

\section{[INSERT FIGURE 3 HERE]}

\section{Conclusion}

In this paper, our endeavour was to contribute to the emerging body of literature that studies the dynamic behaviour of endogenous market structures in dynamic general equilibrium models. We showed that an overlapping generations setting, combined with the idea that entry decisions are made through an occupational choice process, can lead to interesting implications concerning these dynamic patterns. We showed that the intrinsic dynamics of the industry can lead to fluctuations, either though damped oscillations, stochastic sunspot equilibria, or limit cycles. These results represent yet another example on how endogenous forces can cause fluctuations in economic dynamics.

A note of caution merits discussion here, given the fact that that our paper's dynamics are characterised by periodic orbits that may resemble the type of fluctuations we observe in the data. We believe that a better interpretation of our results should entail a correspondence to low frequency waves in industry activity, such as those presented by Comin and Gertler 
(2006) for example, rather than the high frequency fluctuations that are more suitably attributed to the occurrence of short-term business cycles. For this reason, we need to clarify that our analysis in under no circumstances an attempt to invalidate other explanations for the cyclicality of economic dynamics, based on the idea of exogenous shocks - explanations that we actually view as being indubitably important. The main message form our work is that the (medium-term) cyclical behaviour of economies, in addition to being a response to changing economic conditions, may also reflect characteristics that render them inherently volatile. As we indicated at the very beginning of this paper, other authors have asserted the same through their research work, thus offering some momentum to this idea.

The model we presented is simple enough to guarantee a clear understanding of the mechanisms that are involved in the emergence of the basic results, without blurring either their transparency or their intuition. Of course, there is certainly a large scope for getting additional implications by modifying or enriching some of the model's founding characteristics. One obvious direction is to assume that the oligopolistic industry supplies firms with different varieties of capital goods while, at the same time, retaining the important characteristic of endogenous occupational choice. The ensuing process of capital accumulation could set in motion some very interesting implications concerning economic dynamics. Another potential direction is to endogenise the exit rate, perhaps by assuming that firm ownership is bequeathed from parents to children. Again, such a set-up could initiate even richer dynamics, thus it offers a potentially fruitful avenue for future research work. 
Figures (please place the corresponding Figure to the place indicated on the main text)

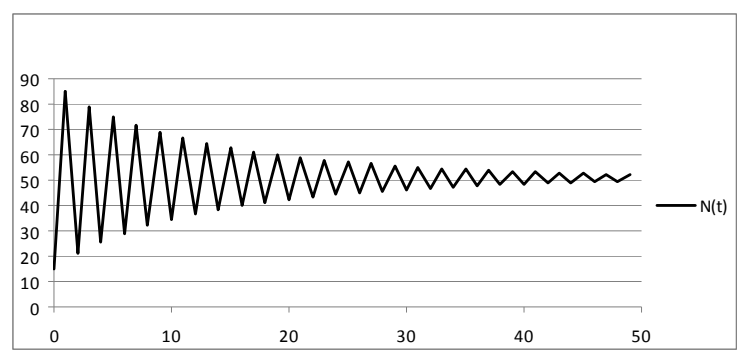

Figure 1. Damped oscillations in market entry

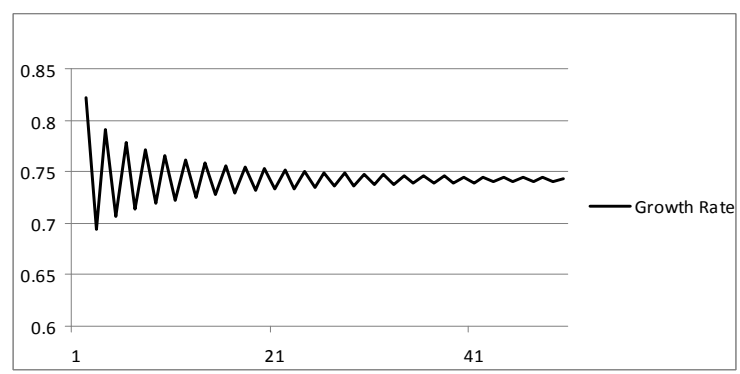

Figure 2. Damped oscillations in output growth 


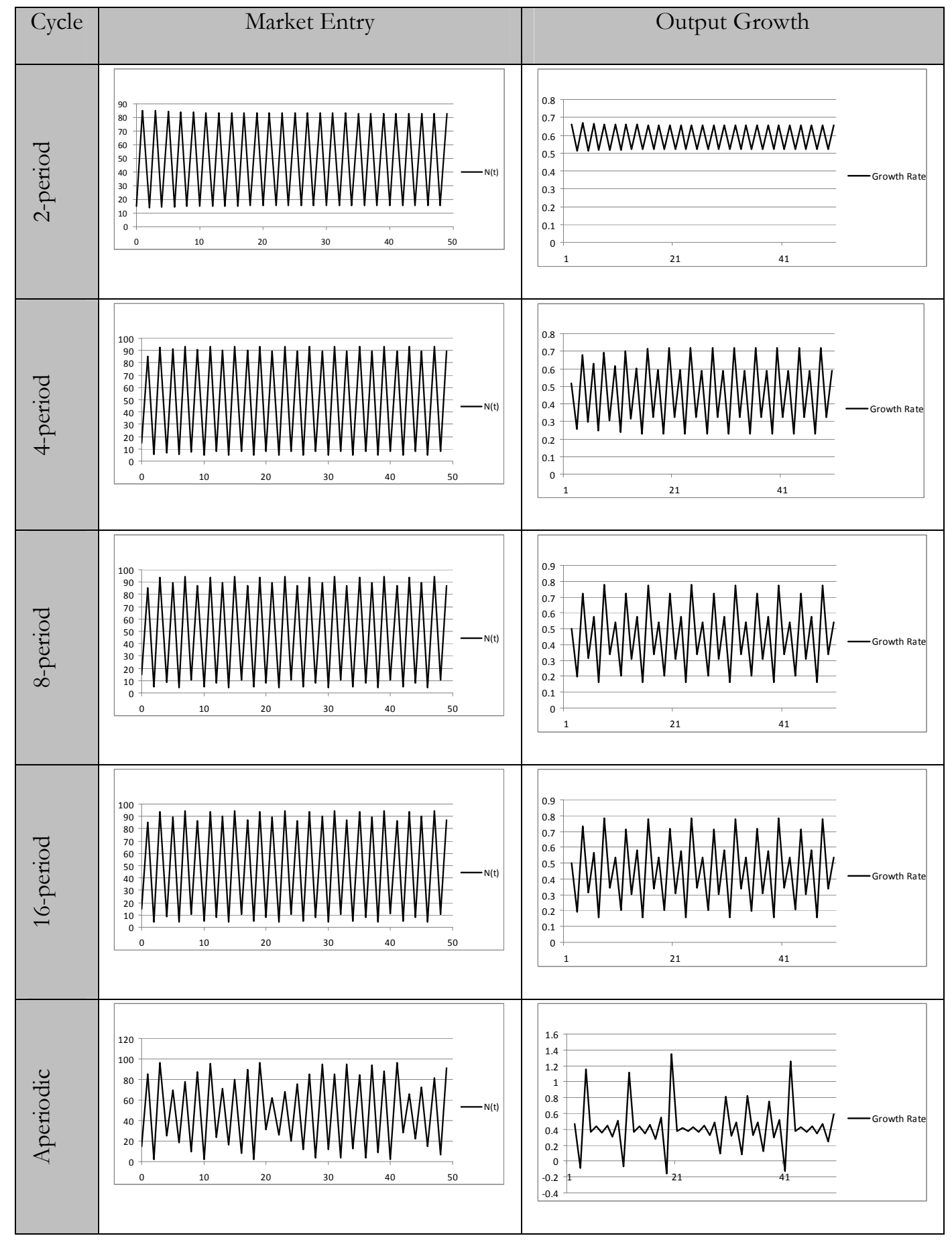

Figure 3. Permanent deterministic cycles 


\section{Appendix}

\section{Proofs of Lemmas 1-4}

The Jacobian matrix associated with the planar system in (40)-(41) is

$$
\left(\begin{array}{ll}
F_{Z_{t}}(\hat{Z}, \hat{N}) & F_{N_{t}}(\hat{Z}, \hat{N}) \\
H_{Z_{t}}(\hat{Z}, \hat{N}) & H_{N_{t}}(\hat{Z}, \hat{N})
\end{array}\right)
$$

where $\hat{N}=\hat{Z}$ is given in (38). Some straightforward algebra with the use of Equations (38), (40) and (41), reveals that the trace $(T)$ and the determinant $(D)$ are equal to

$$
T=F_{Z_{t}}(\hat{Z}, \hat{N})+H_{N_{t}}(\hat{Z}, \hat{N})=-\delta\left\{\theta-\frac{a(1+\delta \theta)}{(1-a)[n-(1+\delta)]}\right\} \text {, }
$$

and

$$
D=F_{Z_{t}}(\hat{Z}, \hat{N}) H_{N_{t}}(\hat{Z}, \hat{N})-F_{N_{t}}(\hat{Z}, \hat{N}) H_{Z_{t}}(\hat{Z}, \hat{N})=\frac{\delta a(1+\delta \theta)}{(1-a)[n-(1+\delta)]},
$$

respectively. Furthermore, the eigenvalues are the roots of the polynomial $\lambda^{2}-T \lambda+D$, i.e.,

$$
\lambda_{1}=\frac{T-\sqrt{T^{2}-4 D}}{2} \text { and } \lambda_{2}=\frac{T+\sqrt{T^{2}-4 D}}{2} .
$$

To ensure the stability of the steady state, we want the eigenvalues to satisfy $\left|\lambda_{1}\right|<1$ and $\left|\lambda_{2}\right|<1$. Given that $\lambda_{1}+\lambda_{2}=T$ and $\lambda_{1} \lambda_{2}=D$, two necessary but not sufficient conditions for stability are $-1<D<1$ and $-2<T<2$. Evidently, the determinant is positive by virtue of (A2); therefore we can use (A2) to find that $D<1$ corresponds to the restriction

$$
n-1>\delta+\frac{\delta a(1+\delta \theta)}{1-a}
$$

Furthermore, note that we can use (A1) and (A2) to get 


$$
T=D-\delta \theta
$$

As we constrain ourselves to $D<1$, Equation (A5) reveals that $T<2$. Therefore, we want to obtain a restriction for which $T>-2$. Using (A5), it can be very easily established that a sufficient (but not necessary) condition for this is given by

$$
\delta \theta<2 .
$$

In addition to the above, we will rule out complex eigenvalues by imposing the parameter restriction that ensures $T^{2}-4 D \geq 0$. Specifically,

$$
\begin{gathered}
T^{2} \geq 4 D \Leftrightarrow \\
(D-\delta \theta)^{2} \geq 4 D \Leftrightarrow \\
D^{2}-2(2+\delta \theta) D+(\delta \theta)^{2} \geq 0 \Leftrightarrow \\
\Phi(D) \geq 0 .
\end{gathered}
$$

It is $\Phi(0)=(\delta \theta)^{2}>0$ and $\Phi(1)=1-2(2+\delta \theta)+(\delta \theta)^{2}<0$ by virtue of (A6). Furthermore, $\Phi^{\prime}=2 D-2(2+\delta \theta)<0$ for $D \in(0,1)$. Hence, for $T^{2}-4 D \geq 0$ to hold we need the restriction $D<\tilde{D}_{\min }$, where $\tilde{D}_{\min }$ is the lowest-valued root of $\Phi(\tilde{D})=0$. We can then use (A2) to establish that

$$
\begin{gathered}
D<\tilde{D}_{\min } \Leftrightarrow, \\
D<2+\delta \theta-2 \sqrt{1+\delta \theta} \Leftrightarrow . \\
n-1 \geq \delta+\frac{\delta a(1+\delta \theta)}{(1-a)(2+\delta \theta-2 \sqrt{1+\delta \theta})} \equiv \Omega(\delta) .
\end{gathered}
$$

However, notice that $2+\delta \theta-2 \sqrt{1+\delta \theta} \in(0,1)$ by virtue of (A6). This implies that the restriction in (A8) ensures that the condition in (A4) is also satisfied.

Now, check that $\delta \theta>2+\delta \theta-2 \sqrt{1+\delta \theta}$. By virtue of (A8), this means that 


$$
n \geq 1+\delta+\frac{\delta a(1+\delta \theta)}{(1-a) \delta \theta}
$$

Consequently, combining (A9) and (A5), we can establish that the trace $T$ is negative, i.e., $T \in(-2,0)$ which, combined with (A3), reveals that both eigenvalues $\lambda_{1}$ and $\lambda_{2}$ are negative. It can be easily established that $\lambda_{2}>-1$, whereas $\lambda_{1}>-1$ holds as long as

$$
\begin{gathered}
\frac{T-\sqrt{T^{2}-4 D}}{2}>-1 \Leftrightarrow \\
2+T>\sqrt{T^{2}-4 D} .
\end{gathered}
$$

Given $T>-2$, we can use the above expression to get

$$
\begin{gathered}
(2+T)^{2}>\left(\sqrt{T^{2}-4 D}\right)^{2} \Leftrightarrow \\
4+4 T>-4 D \Leftrightarrow \\
D+T+1>0 \Leftrightarrow \\
D>\frac{\delta \theta-1}{2} .
\end{gathered}
$$

which holds unambiguously when $\delta \theta \leq 1$. Hence, in this case $0>\lambda_{2}>\lambda_{1}>-1$ holds - a result ensuring that there is convergence to the long-run equilibrium and that it is oscillatory (or cyclical).

Now consider the case where $\delta \theta \in(1,2]$. The condition in (A10) can be written as

$$
n-1<\Xi(\delta),
$$

where

$$
\Xi(\delta) \equiv \delta+\frac{\delta a(1+\delta \theta)}{1-a} \frac{2}{\delta \theta-1} .
$$


Recalling that we are considering values for which $\delta \theta \in(1,2]$, we can determine that $\lim _{\delta \rightarrow(1 / \theta)_{+}} \Xi(\delta)=+\infty$ and $\Xi\left(\frac{2}{\theta}\right)=2\left(1+\frac{3 a}{1-a}\right)<n-1$ by assumption. As long as $\Xi(\delta)$ cuts the $n-1$ line only once, then there is $\tilde{\delta}$ such that $\Xi(\tilde{\delta})=n-1$. Furthermore, note that for $\delta \theta \in(1,2]$ we have $\frac{1}{2+\delta \theta-2 \sqrt{1+\delta \theta}}<\frac{2}{\delta \theta-1}$. Given (A8), (A12) and the assumption $n>1+2\left(1+\frac{3 a}{1-a}\right)$, this implies that $\Xi(\delta)>\Omega(\delta) \quad \forall \delta \leq 2 / \theta$, i.e., the condition in (A8) always holds given our assumptions.

The previous analysis implies that (A11) holds when $\delta<\tilde{\delta}$ and therefore $0>\lambda_{2}>\lambda_{1}>-1$. The steady state $\hat{N}$ is locally stable. However, when $\delta>\tilde{\delta}$ we have $0>\lambda_{2}>-1>\lambda_{1}$ and the steady state $\hat{N}$ is a saddle point. Evidently, at $\delta=\tilde{\delta}$ we have $\lambda_{1}=-1$. Combined with $\lambda_{2} \in(-1,0)$, we can use Theorem 8.4 in Azariadis (1993) to deduce that the dynamical system undergoes a flip (or period doubling) bifurcation so that there exists a 2-period cycle.

\section{References}

1. Azariadis, C. 1993. Intertemporal Macroeconomics, Blackwells.

2. Azariadis, C., and B.D. Smith (1996) Private information, money, and growth: indeterminacy, fluctuations, and the Mundell-Tobin effect. Journal of Economic Growth 1, 309-332.

3. Azariadis, C., and R. Guesnerie (1986) Sunspots and cycles. Review of Economic Studies 53, 725-737. 
4. Baker, N., and A. Agapiou (2006) The production cycles of the Scottish construction industry, 1802-2002. Proceedings of the $2^{\text {nd }}$ International Congress on Construction History, Volume 1.

5. Banerjee, A.V., and A.F. Newman (1993) Occupational choice and the process of development. Journal of Political Economy 101, 274-298.

6. Banerji, S., J. Bhatthacharya, and N. Van Long (2004) Can financial intermediation induce endogenous fluctuations? Journal of Economic Dynamics and Control 28, 22152238.

7. Benhabib, J., and K. Nishimura (1985) Competitive equilibrium cycles. Journal of Economic Theory 32, 284-306.

8. Bilbiie, F.O., F. Ghironi and M.J. Melitz (2012) Endogenous entry, product variety, and business cycles Journal of Political Economy 120, 304-345.

9. Carrasco, R. (1999) Transitions to and from self-employment in Spain: an empirical analysis. Oxford Bulletin of Economics and Statistics 61, 315-341.

10. Chatterjee, S., R. Cooper, and B. Ravikumar (1993) Strategic complementarity in business formation: aggregate fluctuation and sunspot equilibria. Review of Economic Studies 60, 795-811.

11. Colciago, A., and F. Etro (2010) Real business cycles with Cournot competition and endogenous entry. Journal of Macroeconomics 32, 1101-1117.

12. Comin, D., and M.L. Gertler (2006) Medium-term business cycles. American Economic Review 96, 523-551.

13. Devaney, R.L. (2003) An Introduction to Chaotic Dynamical Systems, Westview Press.

14. Dixit, A.K., and J.E. Stiglitz (1977) Monopolistic competition and optimum product diversity. American Economic Review 67, 297-308. 
15. Dos Santos Ferreira, R., and T. Lloyd-Braga (2005) Non-linear endogenous fluctuations with free entry and variable markups. Journal of Economic Dynamics and Control 29, 847-871.

16. Etro, F. (2009) Endogenous Market Structures and the Macroeconomy. Springer.

17. Etro, F., and A. Colciago (2010) Endogenous market structures and the business cycle. Economic Journal 120, 1201-1233.

18. Galor, O. (2007) Discrete Dynamical Systems, Springer.

19. Geroski, P.A. (1995) What do we know about entry? International Journal of Industrial Organisation 13, 421-440.

20. Ghironi, F., and M.J. Melitz (2005) International trade and macroeconomic dynamics with heterogeneous firms. Quarterly Journal of Economics 120, 865-915.

21. Gil, P.M., P. Brito, and O. Afonso (2013) Growth and firm dynamics with horizontal and vertical R\&D. Macroeconomic Dynamics 17, 1438-1466.

22. Grandmont, J.M. (1985) On endogenous competitive business cycles. Econometrica 53, 995-1045.

23. Grandmont, J.M., P. Pintus, and R. de Vilder (1998) Capital-labour substitution and competitive nonlinear endogenous business cycles. Journal of Economic Theory 80, 1459.

24. Kaas, L., and S. Zink (2007) Human capital and growth cycles. Economic Theory 31, $19-33$.

25. Keklik, M. (2003) Schumpeter, Innovation, and Growth: Long-Cycle Dynamics in the PostWWII American Manufacturing Industries, Ashgate.

26. Koellinger, P.D., and A.R. Thurik (2012) Entrepreneurship and the business cycle. Review of Economics and Statistics 94, 1143-1156. 
27. Matsuyama, K. (1999) Growing through cycles. Econometrica 67, 335-347.

28. Mino, K., K. Shinomura, and P. Wang (2005) Occupational choice and dynamic indeterminacy. Review of Economic Dynamics 8, 138-153.

29. Parker, S.C. (2009) The Economics of Entrepreneurship, Cambridge University Press.

30. Rampini, A.A. (2004) Entrepreneurial activity, risk, and the business cycle. Journal of Monetary Economics 51, 555-573.

31. Reichlin, P. (1986) Equilibrium cycles in an overlapping generations economy with production. Journal of Economic Theory 40, 89-102.

32. Sanders, M. (2013) Skill bias in an endogenous growth model: evaluating the case for market size and acceleration effects. Macroeconomic Dynamics 17, 802-829.

33. Woodford, M. (1986) Stationary sunspot equilibria: the case of small fluctuations around a deterministic steady state. Mimeo.

34. Yu, L., P.F. Orazem, and R.W. Jolly (2009) Stopping start-ups: how the business cycle affects entrepreneurship. Department of Economics working paper no. 09014, Iowa State University.

35. Zeng, Z. (2013) Credit frictions and firm dynamics. Macroeconomic Dynamics 17, 14671495. 